\title{
Machine Learning Systems in Clinics - How Mature Is the Adoption Process in Medical Diagnostics?
}

\author{
Luisa Pumplun \\ Technical University of \\ Darmstadt \\ pumplun@is.tu-darmstadt.de
}

\author{
Mariska Fecho \\ Technical University of \\ Darmstadt \\ fecho@is.tu-darmstadt.de
}

\author{
Nihal Islam \\ Technical University of \\ Darmstadt \\ islam@is.tu-darmstadt.de
}

\author{
Peter Buxmann \\ Technical University of \\ Darmstadt \\ buxmann@is.tu-darmstadt.de
}

\begin{abstract}
In a world with a constantly growing and aging population, health is a precious asset. Presently, with machine learning $(M L)$, a technological change is taking place that could provide high quality healthcare and especially, improve efficiency of medical diagnostics in clinics. However, ML needs to be deeply integrated in clinical routines which highly differs from the integration of previous health IT given the specific characteristics of ML. Since existing literature on the adoption of ML in medical diagnostics is scarce, we set up an explorative qualitative study based on a conceptual basis consisting of the technologicalorganizational-environmental framework (TOE) and the healthcare specific framework of non-adoption, abandonment, scale-up, spread, and sustainability (NASSS). By interviewing experts from clinics and their suppliers we were able to connect both frameworks and identify influencing factors specific to the adoption process of ML in medical diagnostics.
\end{abstract}

\section{Introduction}

The ongoing digitalization influences the society and business world, including the healthcare sector as a whole. For instance, the integration of health information technologies (HIT) in clinics such as electronic health records enables significant improvements in therapy, rehabilitation, or diagnostics [21]. However, this new technological opportunities also lead to challenges within clinics: Physicians have to deal with an ever-increasing amount of patient's data created by digitized systems [4]. In addition, clinics currently face major societal issues: The high number of aging-related diseases caused by demographic change is - for example-further increased by the global COVID-19 pandemic crisis, so that the resources of medical personnel become progressively strained [27]. Artificial intelligence (AI) as the "science and engineering of making intelligent machines, especially intelligent computer programs" [31:2] could help solving such challenges and makes it possible to technically solve tasks that were previously reserved for human intelligence [38]. Especially, machine learning (ML) as a subfield of AI is currently one of the most rapidly growing technological opportunities. Thus, in this research paper, we focus on ML, which enables information systems (IS) to improve themselves automatically through training experience $[8,23]$. ML systems have the enormous potential to process complex patient data (e.g. medical images or text data) effectively, find hidden patterns in symptoms, and link them to possible diseases. In this regard, the use of ML systems for diagnostics could enable more profound and efficient diagnoses and could thus be decisive for life or death [18, 42]. However, ML systems also pose challenges, which prevents a wide-spread implementation in clinics so far [24]. This is particularly the case as ML systems differ from other HIT as they are able to adapt their behavior over time, operate as black boxes, derive results statistically, and can thus lead to erroneous decisions [8].

IS research has recently begun to investigate the chances and challenges of ML in healthcare. For example, it has been analyzed what ML systems can contribute to improve medical processes and, in particular, medical diagnostics [e.g. 19, 25]. Furthermore, technical research is being done to demonstrate the feasibility of ML systems' application in medicine [e.g. 3, 29]. However, prior studies have not yet contributed sufficiently to an understanding of clinics' adoption process of ML systems. In that regard, our research aims to identify: Which specific factors influence the readiness of clinics to adopt $M L$ systems and subsequently implement such systems for medical diagnostics? To answer this question, we conducted a qualitative study based on interviews with experts working for clinics or their suppliers. In order to sort our key findings, we refer to the technologicalorganizational-environmental (TOE) framework and the framework of non-adoption, abandonment, scaleup, spread and sustainability (NASSS) as a conceptual basis $[12,17]$. 


\section{Theoretical background}

ML systems are based on algorithms capable of extrapolating patterns from data [39]. The acquired patterns can then be applied to new data in order to make, for example, classifications. As a consequence, ML systems are able to solve tasks without receiving explicit instructions but by learning from training examples. These examples can either be labelled (e.g. symptoms and related condition) or without any annotation (e.g. symptoms), resulting in a respective supervised or unsupervised ML problem [8]. Due to the data-based learning approach, an inherent property of ML systems is that they can be adapted to new data and evolve over time if being retrained [39].

Reaching from prediction of patient traffic in clinics to support of therapies: ML systems can help solving various problems in medicine [42, 43]. However, one of the most prominent areas of application in research and practice are medical diagnostics [e.g. 35, 41]. In this context, ML systems (and especially deep neural networks) can help to identify patterns in medical data (e.g. in medical scans, pathology slides, electrocardiograms) and sort possible conditions according to their likelihood [18, 44]. A distinction can be made whether ML serves to take over entire areas of responsibility from doctors or to support them in their decision-making process. In the near future, ML systems will mainly be used as an intelligent decision support rather than to automate medical diagnostics fully [e.g. 18, 25]. In this sense, current practical examples such as Isabel Health (an ML based symptom checker) show that more and more of such assistive ML systems are presently finding their way into clinics [46]. These systems raise the hope of making medical diagnostics faster, more efficient, and consistent and thus more valuable since they are able to compare patients' data with a database that is larger than any physician's experience [18, 42]. However, the introduction of ML systems in clinics also poses major challenges as these systems highly differ from conventional HIT. ML systems learn from high volumes of data, instead of being explicitly programmed [39]. It is therefore imperative to share data across clinics to enable profound training of the ML system [18]. Provided that ML systems are based on appropriate data, they are able to prepare high stakes diagnostic decisions (e.g. by suggesting possible conditions) [25]. ML systems derive these solutions based on statistical methods, which leads to several consequences: Modern ML systems are not only becoming increasingly opaque, they also never lead to $100 \%$ accuracy [8, 25]. These properties are particularly problematic in a medical setting where patients' lives depend on a profound diagnosis and the correct functionality of the ML system should be ensured at any time [25]. Given the specific characteristics of ML systems, a wide-spread adoption in clinics has not been achieved so far, requiring more detailed investigation by research [24].

In order to gain an overview of existing literature regarding the adoption process of ML systems in clinics, a systematic literature review was conducted and published by the authors [37]. Looking at the articles identified, it becomes clear that most of the publications do not include primary empirical studies to identify factors influencing the adoption process of ML systems in clinics. Furthermore, none of the publications based their research on pertinent theories in order to develop a holistic understanding of the adoption process in clinics. Even though, ML systems are highly relevant in medicine, there is thus a lack of empirical and theoretically profound research. Against this background, an investigation of the challenges related to the adoption process of ML systems in clinics is desirable. To gain deeper insights into this research field, it is important to differentiate that the adoption process of innovations in organizations consists of two main phases comprising: (1) The initial readiness for a technology and (2) its subsequent implementation within the organization [50]. Both steps are highly relevant to the success of a technology within an organization and should be considered as dependent from each other [45]. The two-stage adoption process is a complex, multi-dimensional process that is difficult to represent using a single theory. In line with the recommendations of Mayer and Sparrowe (2013), we therefore base our research on "two theories to address what neither theory could [explain] independently" [30:917]. We thus utilize two frameworks to investigate the factors that influence a clinic's process from (1) the first readiness to (2) the implementation of ML systems. Accordingly, we chose to employ both (1) the TOE framework developed by DePietro et al. (1990) [12] and (2) the NASSS framework [17] as a conceptual basis.

The TOE framework is widely used in IS research to understand the readiness of an organization to adopt a new technological innovation [e.g. 1, 47]. In this regard, it considers three domains: The technological, organizational, and the environmental context. Each context, in turn, comprises several factors that specify on the considered domain. Those are for example the characteristics of the investigated technology (technological context), the existing infrastructure within an organization (organizational context), or governmental regulations concerning a technology (environmental context) [12]. In the last few years, clinics are increasingly faced with technological change and have to decide which technologies are 
relevant to them [2]. In that regard, several studies have used and adapted the TOE framework to explain the factors that influence the readiness of a clinic to adopt an HIT, although the framework was not originally established for the healthcare industry [e.g. $22,28]$. Thus, we consider the theoretical framework to be a useful starting point to investigate what enables clinics to prepare for the adoption of ML systems.

Presently, adoption research in health informatics started to look beyond the mere readiness and towards the implementation phase after the adoption actually took place [17]. In this context, ML systems own highly specific characteristics that will necessitate a significant change in the organization's structure and working routines in the long run $[8,25]$. Therefore, the implementation phase after the first readiness to adopt is just as decisive for the success of ML systems in clinics as the previous one. To capture this, we included the NASSS technology implementation framework as a second conceptual basis. NASSS has primarily been developed for the healthcare context by combining established health- and social care frameworks and can be used to analyze the implementation phase of an HIT. It includes seven domains such as the condition to be diagnosed and treated, the demand- and supply-side value proposition associated with an HIT, and the adopter system consisting of patients, their relatives and medical staff. Furthermore, it explicitly conceptualizes on the embedding and adaptation of the HIT within a clinic over time [17]. In summary, the factors of NASSS help to complement the TOE framework to account for the whole adoption process of a HIT in clinical processes.

Since ML systems differ significantly from existing technologies and it is not sufficient to rely on either the basic TOE or NASSS framework [49], we seek to combine, adapt, and extend both frameworks in the following to gain a profound understanding about the specific factors that enable clinics to put ML systems into clinical practice.

\section{Qualitative research methodology}

Our overarching goal was to identify the factors that are specific to the adoption process of ML systems in clinics and are not yet sufficiently covered by existing theories. We thus followed the steps of directed content analysis in order to extend the established conceptual framework based on TOE and NASSS. In that regard, we used both frameworks as a starting point that were integrated, adapted, and expanded taking into account the qualitative data [20].

To analyze and understand the highly complex process from a first readiness to a routine use of ML systems, an in-depth analysis was necessary. We therefore employed a qualitative approach to "see the world through the eyes of the actors doing the acting" [16:17] and conducted interviews with highly involved experts $(\mathrm{N}=15)$. We formulated a semi-structured interview guideline to lead the conversation and to ensure that all relevant questions are posed. Due to the qualitative approach, the guideline was kept open and flexible to allow adaptations to the respective interview partner, one's position and knowledge base [33].

The qualitative data were collected from May to October 2019 within Germany. In order to identify suitable participants, we have searched for experts in social networks, on clinic websites, and at relevant conferences on ML in medicine. Qualified interviewed experts $(\mathrm{N}=15)$ were chosen, who have detailed knowledge of clinical processes, experience with ML systems, and are involved in the respective decisionmaking processes [7]. We consider the additional supplier perspective to be particularly useful to triangulate the data [9]. The various experts are clinics' managers, physicians, and managers of diagnostic HIT suppliers. While three of the interviewed experts were physicians, six hold a hybrid position (i.e. physicians with additional leadership responsibilities), and six were full-time managers with medical education. As shown in Table 1, different medical disciplines were considered in the interviews (e.g. radiology, pathology, internal medicine) in order to allow for different perspectives on medical diagnostic processes (e.g. interpretation of medical scans, pathology slides, electrocardiograms) and obtain more generalizable results [5]. All experts had in common that they had prior knowledge on ML systems due to research work, pertinent projects, or product development processes.

Table 1: Study participant overview

\begin{tabular}{|l|l|l|l|}
\hline \multicolumn{2}{|l|}{ Position } & Specialty & Exp. in yr. \\
\hline $\begin{array}{l}\text { Clinics (C): Key informants of clinics } \\
\text { *: } \text { Physician with leadership responsibilities }\end{array}$ \\
\hline 01 & Physician & Radiology & 3 \\
\hline 02 & Physician & Radiology & 15 \\
\hline 03 & Physician & Radiology & 8 \\
\hline 04 & Physician* & Neuro-radiology & 9 \\
\hline 05 & Physician* & Internal medicine & 19 \\
\hline 06 & Physician* & Internal medicine & 35 \\
\hline 07 & Physician* & Pathology & 18 \\
\hline 08 & Physician* & Radiology & 37 \\
\hline 09 & Physician* & Gynecology & 40 \\
\hline 10 & CTO & Cardiology & 8 \\
\hline 11 & CTO & Biomedicine & 20 \\
\hline 12 & Director & Internal medicine & 12 \\
\hline HIT Supplier (S): Key informants of clinics' HIT \\
supplier companies
\end{tabular}


Participants work for several clinics and HIT suppliers (i.e. nine different clinics, three HIT suppliers). While three clinics are privately financed, the others are public. Furthermore, all clinics and suppliers are currently running projects related to ML. On average, each expert interview lasted 49 minutes and took place in private space. The interviews were audio recorded and transcribed after mutual agreement. In two interviews only, notes were taken as the participant refused audio recording. The transcripts were analyzed with the help of NVivo 12 software. We applied an iterative multi-cycle coding process that is in line with qualitative content analysis and that consisted of two coding cycles, between which we moved back and forth [40]. The first cycle comprised three different types of coding: Using attribute coding enabled us to receive descriptive information concerning the participant. Hypothesis coding was employed to consider the prespecified conceptual framework (i.e. TOE and NASSS) and to examine the suitability of existing factors regarding the adoption process (e.g. clinic's size). In contrast, the descriptive coding approach allowed us to identify new aspects that go beyond the conceptual framework by disregarding formerly identified factors. Since the coding procedure during the first cycle has led to a large number of factors, we used pattern coding within the second coding cycle to pull together the codes into a smaller number of constructs [40].

During the research process, we employed several practices to obtain rigor and trustworthiness. To begin with, we defined a clear research question and conceptual framework that we used as input for our research design. Furthermore, we followed a theoretical sampling approach by iterating between data collection and analysis until theoretical saturation was reached after the $15^{\text {th }}$ interview [15]. The resulting amount of interviews is comparable to other qualitative studies in IS (healthcare) research [e.g. 6, 19]. Besides, a multi-researcher triangulation took place. In that sense, coding was intensively discussed between the authors during the data analysis [9]. We also decided to include the voice of participants and thus quoted directly from the interviews while presenting our findings [10:182].

\section{Results and discussion of findings}

As diagnostic procedures can differ within the different medical specialties, the data analysis focuses on common factors that affect the adoption of ML systems for diagnostics in clinics and can be derived across all disciplines. The key findings are structured according to the TOE and NASSS framework in order to describe the holistic adoption process of $\mathrm{ML}$ systems. An integrative overview of all these factors can be seen in Figure 1. In this regard, the first three propositions (abbreviated: P) refer to clinic's readiness to adopt ML systems for diagnostics, while P4-6 apply to the implementation phase. However, P7 shows the relevance of patient data for both adoption phases.

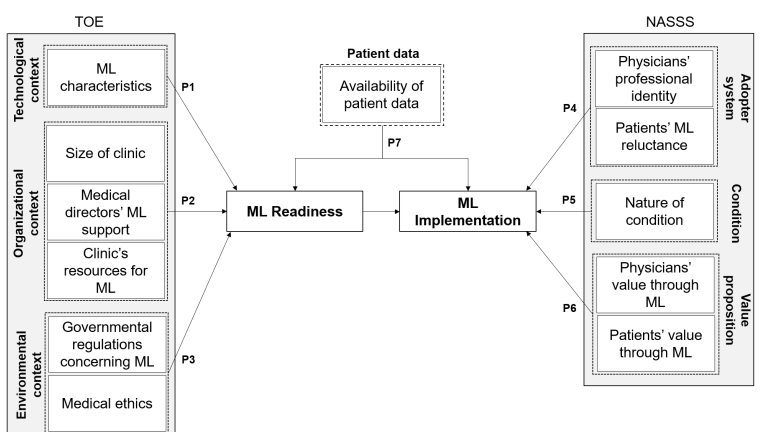

Figure 1: Integrative overview of the findings

In the following, we present and discuss the results of our data analysis. For this purpose, we structure our findings according to the domains technological, organizational, and environmental context as well as the adopter system, condition, value proposition, and the stand-alone domain patient data.

Technological context. The characteristics of a technology are a factor that is already considered within the original TOE framework [12]. Nevertheless, as outlined earlier, ML systems encompass several highly specific characteristics, that cannot be compared to other technologies. Therefore, the existing general factor "characteristics" is not sufficient to capture the properties of ML and has to be specified further.

As one sub-factor of $M L$ characteristics, the interviewees point out the 'lack of transparency' of ML systems as a major obstacle for clinic's readiness to adopt ML systems. ML systems based on neural networks can consist of multiple processing layers and up to millions of numerical weights hampering the comprehensibility of ML systems to humans [8]. Especially in high-stakes decision-making processes such as medical diagnostics, this can lead to major issues. In this context, the experts state that physicians need to know exactly, which are the critical features considered by ML systems and how identified patterns lead to conclusions to be able to assess the ML system's recommendation and suggest an appropriate therapy. One of the experts underlines this aspect: "You will never make these existential decisions dependent on a black box, where it is not possible to understand what led to the recommendation" (C-06). Another sub-factor of ML characteristics is the "ability to adapt' their functioning if being retrained on novel data. This can either become relevant when the ML 
system is transferred to another context (e.g. another clinic) or needs to be retrained after some time as for example new medical research results are gained or patients' demographic structure shifts. Clinics thus have to deal with an opaque system that is able to change its reasoning, making the outcome of a ML system unpredictable. Accordingly, experts see the adaptability of ML systems as another factor that has to be addressed by clinics (S-01, S-03). In order to prepare for the adoption of ML systems, clinics need to have a clear strategy in place on how to cope with the opacity and adaptability of self-learning ML systems. We thus state our first proposition:

P1: The characteristics of ML systems (i.e. lack of transparency, adaptability) will impede the readiness of clinics to adopt ML systems.

Organizational context. Looking at the organizational context domain, three factors emerged during the interviews: size of clinic, medical directors' $M L$ support and clinic's resources for $M L$.

The size of a clinic is similar to an existing TOE factor that was also considered relevant in the context of ML systems. In this sense, experts emphasized that small clinics have usually less resources compared to large clinics, which could hamper their readiness to adopt ML systems (C-11). In addition, larger clinics care for a larger number of different patients and thus have access to higher amounts of patient data which is needed to train ML systems appropriately (S-01).

Furthermore, experts state that the first decision to adopt ML systems for diagnostic processes needs to be supported by clinic's medical directors to achieve financial and non-financial support for the new technology (C-03). In this regard, ML systems for medical diagnostics affect the core business of clinics and thus have a strategic relevance [49]. As medical directors develop the clinic's strategy, they are responsible for paving the way regarding the readiness of clinics to adopt ML systems. This is in line with prior research that states the significance of medical directors' support regarding the readiness of clinics for strategically relevant HITs [28, 47].

One of the most frequently stated factors within the organizational context are clinic's resources to get ready to adopt ML systems. This factor incorporates three sub-factors which are either in line with the original TOE framework (i.e. 'clinic's technical infrastructure') or newly added (i.e. 'financing structure', 'expertise in medicine and data science'). In line with existing literature [34], some of the experts report that clinics frequently rely on a wide range of clinical legacy systems, which are often proprietary to the suppliers, not connected, and based on obsolete hardware: "The primary challenge [...] is that the clinic usually consists of [...] million proprietary systems that are not connected" (C-01). However, experts emphasize the importance of a highperformance technical infrastructure that can efficiently access data from different sources to achieve readiness for ML systems' adoption (C-01, C07). Therefore, clinic's technical infrastructure could pose a major challenge for the introduction of ML systems.

The interviewed experts furthermore point out the problem of the current financing structure of clinics, which leads to strict budgetary constraints (C-09). In this regard, an interviewee states that one part of their budget is assigned to daily costs such as medication. The other part of the budget can be used to purchase large-scale medical equipment like X-ray systems. Thus, the development and set-up of ML systems is not covered by either of the two parts and no specific ML budget can be claimed (C-06).

Beyond that, there is a lack of personnel in clinics having both expertise in medicine and data science: "The shortage of medical specialists hits us twice as hard. We feel this at the medical professional side [...], but it is also very apparent at the technical side" (C10). Both fields of knowledge are regarded as highly important for the readiness to adopt ML systems by the experts (C-01, C-10, S-02). While a medical background can help to identify relevant training data or to assess the suggested conditions of the ML system, technical expertise is needed to realize and train ML systems, as presently, nearly no out-of-the-box ML systems exist for the application in medicine, requiring clinics to develop ML systems by themselves (C-01, C10, S-02). Therefore, specific expertise in the field of data science is needed in addition to the medical understanding in order to develop and set up ML systems in clinics. In sum, we propose:

P2: A larger clinic size, medical directors' $M L$ support, and the availability of resources for $M L$ (i.e. technical infrastructure, ML budget, expertise in the field of medicine and data science) will facilitate the readiness of clinics to adopt $M L$ systems.

Environmental context. With regard to the environmental context there are two relevant factors influencing the readiness to adopt ML systems: governmental regulations concerning $M L$ and medical ethics. Governmental regulations are a factor already known from the original TOE framework. Nevertheless, the interviews revealed some particularities that are not covered by the general concept and are described below. Medical ethics is a factor that is not captured by TOE so far, but is identified through our study.

In the field of medicine there are several governmental regulations which must be taken into consideration when adopting ML systems. The 
following sub-factors could be identified: 'medical approval of ML systems', 'accountability', and the 'protection of sensitive personal data'.

The experts draw attention to the fact that HIT offered on the market and used in clinics are subject to several laws. That includes the need for medical approval conducted by legal authorities or HIT suppliers themselves (C-03). In the USA, the Food and Drug Administration (FDA) is responsible for the admission of medical products. In Europe, the HIT suppliers themselves need to perform a conformity assessment procedure, e.g. based on the Medical Device Regulation (MDR) [14, 32]. As mentioned before, most ML systems are currently being developed by clinics themselves and have not undergone any approval process (C-03). However, legal approval of ML systems is not trivial, as the system can learn from new experience and adapt themselves as described above: "It is not obvious how evidence can be obtained for an [ML] model that differs significantly at the beginning, middle, and end of the study. If you want to approve a medical device today, you have to describe the intended use in detail" (S-01). This legal gap is also addressed by an official statement of the FDA that proposes a change to the current regulation to be able to approve adaptable ML systems [14]. A comparable position of the EU does not exist. Therefore, legal ambiguities could represent a hurdle for clinics to decide on the adoption of ML systems for diagnostics.

In addition to the medical approval of a ML system, there is the question of accountability for diagnoses. The experts interviewed indicated that it is questionable who takes over responsibility, if the diagnosis that was prepared by an ML system is inaccurate (C-04, C-10). It is also unclear who can be held liable - the HIT provider, the clinic, or the physician who is providing the medical diagnosis. An expert underlines this aspect with the following words: „Then there are certainly [...] legal problems, for example: who is responsible for the interpretation and possibly wrong results of the ML model?" (C-10). According to the current state of the art, ML systems cannot be held responsible for their output, since a registered physician is always obliged to validate and interpret the system's results and to perform the final diagnosis (C-12). However, it would ease the decision of clinics to opt for ML system if there was a legal specification-especially if ML systems are increasingly able to automate steps of sensitive processes as diagnostics (C-10, C-11).

Another sub-factor which could be identified as relevant for the initiation and adoption of ML systems for diagnostics is the protection of sensitive personal patient data. Patient data are considered as highly sensitive and are under special protection by national and international laws (C-02, S-02). For example, the General Data Protection Regulation (GDPR) in Europe only permits the processing of health data, if the patient explicitly accepts or if the clinic can provide particular reasons [13]. Thus, the respondents emphasize clinics' concerns to obtain the necessary patient data to train the ML system (C-02, C-08).

Using ML systems for diagnostic processes fueled medical ethical concerns among the interviewees. On the one hand, ML systems are able to improve the efficiency and effectivity of diagnostics (C-11, C-12, $\mathrm{S}-02$ ). On the other hand, the suggestions provided by ML systems are deduced based on statistical methods recognizing patterns in patient data that can be biased (C-11). Furthermore, experts claimed that ML systems that are fed with patient data could determine whether a patient tends to develop a disease. This type of medical application would contradict the "patient's right not to know" (C-11). Summarizing these remarks, we set up the proposition:

P3: Uncertainties in governmental regulations, strict requirements for the protection of sensitive patient data, and existing medical ethics will impede the readiness of clinics to adopt $M L$ systems.

Adopter system. The NASSS framework suggests that the successful implementation of ML systems is strongly influenced by the individuals who are supposed to use the system or affected by their suggestions. In this context, two ML specific factors turned out to be relevant according to the interviews, which further specify the factor: threat to physicians' professional identity and patients' ML reluctance.

Since ML systems have the ability to solve tasks that were previously performed by humans, physicians might feel interchangeable in their job (C-03, C-05, S03). ML systems are trained on large sets of data, which exceed the experience of any single physician, setting new standards for medical diagnostics. In this regard, most experts are concerned that physicians could reject ML system for their daily work: "As a doctor who may have ten or 20 years of experience [...], would I like to be taught by a machine [...]? " (S03 ). These concerns have recently found its way into pertinent research, demonstrating the relevance of the topic [e.g. 25].

The majority of the interviewees state the importance of patients' view on the use of ML systems for medical diagnostics. Even though a physician is still involved in the decision-making process, patients might refuse the use of an ML system as the physician may be influenced by suggestions for possible conditions that are derived statistically and could be affected by biases. Furthermore, personal sensitive patient data have to be processed in order to gain 
results. Therefore, experts state patients' acceptance of ML systems as highly relevant for the implementation (C-02, C-04, C-10). We thus conclude:

P4: The threat to the professional identity of physicians and patients' reluctance towards ML systems will impede the implementation of $M L$ systems in clinics.

Condition. As specified within the NASSS framework, the patient's nature of condition impacts the applicability of a technology. This does not only hold true for conventional HIT but is also stated for ML systems by the interviewed experts (C-02, C-07). Therefore, the nature of the condition and the according medical screening decides if ML can be applied to support a diagnostic process at all. Even though ML is considered a general-purpose technology [8], the experts see difficulties to use ML systems, for example, to recognize patterns of conditions in images of organs, which either differ vastly from person to person (e.g. female breast) or are in motion continuously (e.g. human bowel) (C-02). Thus, the use of ML systems will initially be limited to certain conditions:

P5: The limited applicability of ML systems for the diagnosis of specific conditions will impede the implementation of ML systems in clinics.

Value proposition. The value proposition is the third domain of the NASSS framework, we were able to concretize by analyzing the interviews. According to the experts, the implementation of ML systems could result in the creation of value for both the physicians and the patients (C-03, C-08, C-10).

Implementing $\mathrm{ML}$ systems in their daily work enables physicians to improve the effectivity and efficiency of their diagnostics since they can base their decision on a broad data base that is evaluated within a few seconds (C-12): "If you have the choice among a pathologist who has already looked at 10,000 cuts [...] compared to one who has created only 500 findings, whom would you chose? But [...] AI has not only 10,000 but 500,000 findings in memory" (C-06). In this regard, ML systems that are for example based on image recognition algorithms can surpass the ability of the human eye to capture details and patterns in X-rays [3]. If used as a second opinion, ML system thus increase the quality of physicians' work (C-02, C-09).

Also, patients could directly benefit from a decision that is faster and more informed if physicians use ML systems for diagnostics (C-08, C-12). We thus propose: P6: The additional value for physicians and patients created through ML will facilitate the implementation of ML systems in clinics.

Patient data. During the interviews, nearly all experts stated the availability of patient data as crucial for both the readiness of a clinic to adopt and implement ML systems for diagnostics. In this regard, patient data has to be available to develop and train the ML system in the first place and subsequently retrain it during use. This factor comprises certain sub-factors which are described in the following.

According to the experts, most of the clinics generate high volumes of patient data through their daily diagnostic processes (C-03, S-01), which is basically positive since an appropriate 'amount of data' are needed to train ML systems [8].

However, interviewing the experts revealed that medical patient data are usually provided in a variety of 'proprietary data formats' since many disparate clinical legacy systems from different suppliers have to interact in order to enable physicians to provide laboratory tests, diagnostic images (e.g. X-rays), or clinical notes. These proprietary data formats are often difficult or impossible to convert, making the generation of consistent formats highly problematic (C-03). The problem of differing data formats in clinics has already been recognized outside the ML context, e.g. when adopting cloud solutions in healthcare environments [e.g. 48]. Nevertheless, it is particularly critical for the introduction and use of ML systems that the patient data can be processed in order to be able to train and retrain the system. Although first research has been conducted to allow for the transformation of different medical data types in one format [26], most clinics have not yet been able to implement unified standards for patient data in order to enable the processing and analysis by ML systems.

Furthermore, patient data are often stored in 'unstructured file types' such as image, text, or video. Experts raised the concern that physician letters are frequently written in free text formats, which are filled with synonyms and can be individually interpreted. For example, personal formulations are used, such as the description of a tumor's size as comparable to a walnut (C-01). Thus, the patient data are not only hard to harness and has to be transferred in a machine-readable format first (C-03), it also lacks common quality standards for patient data, impeding the extraction of generalizable patterns through ML. Clinics aiming to adopt ML systems to support their diagnostics should therefore establish a common language that physicians apply when creating free texts. Such efforts are already being driven by some national initiatives (C-12).

Moreover, it has been strongly emphasized by the experts, that clinics, which want to use patient data to train ML systems, need to anonymize the sensitive data before processing it through an ML system (C-11). However, 'anonymizing data' might remove valuable information, which could be important regarding the final diagnosis. For instance, information about a person's residence could facilitate a diagnosis if a 
disease is more prevalent regionally $(\mathrm{C}-11)$. Therefore, it is necessary for clinics to find ways to anonymize patient data without losing relevant correlations. First steps are already being taken in technical research to balance the protection against the quality of sensitive data effectively [e.g. 36].

In addition, there is no 'basic truth' for a healthy patient as the human body is a highly complex, not fully understood system. Therefore, no standard for an entirely healthy human can be determined as every medical examination could be influenced by the selection of medical measures, undiscovered diseases, or environmental conditions (S-02). In this context, analyzing the quality of patient data is problematic: " $A$ standard for 'what is healthy' is not defined" (S-02).

According to the experts, the selection of the right training data is especially important in a healthcare context, since wrong diagnoses may have an impact on patients' lives. This leads to another aspect of patient data, which has to be considered: their 'representativeness'. Patients of clinics vary in many aspects - from an outer perspective (e.g. age, gender, hair color) as well as from the inner functioning (e.g. size of organs, blood values). If ML systems are predominantly trained based on a demographically or regionally distorted database, false conclusions could be drawn by the system. In this context, an expert raised the example of a ML system supporting the detection of skin melanomas, which is mainly trained on a sample of patients with a similar phenotype. Therefore, this pre-trained ML system cannot be easily transferred to patients of other ages or with other skin pigmentation (C-01). As training data for supervised learning need to be labelled by humans, the same could be said regarding the expertise and working philosophy of physicians, which could be highly heterogeneous depending on physician's knowledge state and working environment (C-07, C-10).

Another aspect that influences the availability of ML systems in clinics is the digitization of patient data'. Even though high volumes of data are generated, many processes in clinics are still paper-based impeding the availability of patient data in a digitized form: "Data are often not digitized, much is still in paper files, not structured, which means that the data availability is really extremely [...] poor" (C-03). This observation is in line with prior research concerning clinics who are lagging behind at using digitized technologies [e.g. 21]. As a consequence, the interviewed experts see the integration of an electronic medical record system as a prerequisite for the application of ML systems (C-12, C-03).

Additionally, the availability of patient data is limited due to difficulties of 'patient data sharing'. Although some experts state that their clinics already have some special data networks in place, most healthcare organizations are still not connected. To enhance the availability of patient data in order to train ML systems, more secure internal (within clinic) and external data networks (e.g. clinic-to-primary care) should be established (C-03).

The availability of patient data is not only a factor that decides on the readiness of a clinic to adopt $\mathrm{ML}$ systems, but must also be guaranteed during implementation phase in order to feed and retrain ML systems. Therefore, we identify the availability of patient data as an overarching factor as it influences both the readiness and implementation phase:

P7: The availability of large volumes of digitized patient data (that are structured, uniformly formatted, anonymized, and representative) will facilitate the readiness of clinics to adopt and the implementation of ML systems in clinics.

\section{Conclusion}

ML has an impact on all areas of human life including the healthcare system. In this regard, ML systems offer the opportunity to make diagnostics more efficient and informed. However, in order to harness ML for such an application, clinics need to deeply integrate ML systems into their clinical practice-a challenge that most clinics have not yet been able to overcome [24]. Since clinics own highly individual, human-oriented processes, it is crucial for IS researchers to reflect on this specific context [11].

The prior research is lagging behind to provide empirically proven factors that influence the integration of ML systems in clinics for diagnostic processes. To address this shortcoming, we set up a qualitative study and structured our findings in an integrated overview based on the frameworks TOE and NASSS. Before we discuss our contributions to theory and practice, it is necessary to clarify the limitations of this study. Since we pursued a qualitative approach, our results are based on the expertise of 15 interviewees. In order to counter potential problems of generalizability, we have applied various criteria to ensure rigor and trustworthiness of our study (e.g. theoretical saturation, triangulation, inclusion of multiple medical disciplines). Nevertheless, it might be interesting for further research to perform a quantitative study to verify the stated propositions and validate the proposed framework (i.e. P1-P7). Furthermore, the interviews were conducted in Germany only. Since the healthcare systems vary across nations, interviewing experts from other regions could lead to differing results. In addition, the rapid development of increasingly advanced ML algorithms could lead to systems, which are able to not only 
augment but automate diagnostic processes. Investigating automated diagnostics could produce different findings, even though the results obtained in this study could provide first indications. Nevertheless, our study makes several important contributions. To begin with, it could be shown that the TOE and NASSS framework can be applied, but have to be integrated and expanded in order to explain the full adoption process of ML systems for diagnostics in clinics. On this basis, we are the first to provide an integrative overview of readiness and implementation factors regarding ML systems in clinics. The overview includes three propositions that affect the readiness of a clinic to adopt ML systems and three that impact the subsequent implementation phase to put these systems into clinical use. Availability of patient data is found to be overarching as it influences both the readiness and implementation phase. Therefore, we contribute to adoption research in health informatics, which has recently called to look beyond the mere readiness to adopt HIT in healthcare organizations and to emphasize its subsequent implementation [17]. In addition, our study holds important practical implementations. In this regard, the key findings could guide medical directors of clinics aiming to integrate ML systems within their diagnostic processes. For example, clinics are still lacking strategies to implement unified patient data formats, even though research efforts in this area already exist. Thus, our study could lay a foundation to avoid pitfalls that could occur during the readiness or implementation phase of ML systems in a medical environment.

\section{References}

[1] Aboelmaged, M.G., "Predicting e-readiness at firm-level: An analysis of technological, organizational and environmental (TOE) effects on e-maintenance readiness in manufacturing firms", International Journal of Information Management 34(5), 2014, pp. 639-651.

[2] Agwunobi, A., and P. Osborne, "Dynamic capabilities and healthcare: A framework for enhancing the competitive advantage of hospitals", California Management Review 58(4), 2016, pp. 141-161.

[3] Akcay, S., M.E. Kundegorski, C.G. Willcocks, and T.P. Breckon, "Using deep convolutional neural network architectures for object classification and detection within $\mathrm{x}$ ray baggage security imagery", IEEE Transactions on Information Forensics and Security 13(9), 2018, pp. 22032215.

[4] Bardhan, I., H. Chen, and E. Karahanna, "Connecting systems, data, and people: A multidisciplinary research roadmap for chronic disease management", MIS Quarterly 44(1), 2020, pp. 185-201.

[5] Benbasat, I., D.K. Goldstein, and M. Mead, "The case research strategy in studies of information systems", MIS
Quarterly: Management Information Systems 11(3), 1987, pp. 369-386.

[6] Blankenhagel, K.J., M.-M. Theilig, H. Koch, A.-K. Witte, and R. Zarnekow, "Challenges for Preventive Digital Stress Management Systems - Identifying Requirements by Conducting Qualitative Interviews", Proceedings of the 52nd Hawaii International Conference on System Sciences, (2019), 3810-3819.

[7] Bogner, A., B. Littig, and W. Menz, "Introduction: Expert interviews - An introduction to a new methodological debate", In A. Bogner, B. Littig and W. Menz, eds., Interviewing Experts. Palgrave Macmillan, London, 2009, 113.

[8] Brynjolfsson, E., and T. Mitchell, "What can machine learning do? Workforce implications", Science 358(6370), 2017, pp. 1530-1534.

[9] Carter, N., D. Bryant-Lukosius, A. Dicenso, J. Blythe, and A.J. Neville, "The use of triangulation in qualitative research", Oncology Nursing Forum 41(5), 2014, pp. 545547.

[10] Creswell, J.W., Qualitative inquiry and research design: Choosing among five traditions, Sage, Thousand Oaks, California, USA, 2007.

[11] Davison, R.M., and M.G. Martinsons, "Context is king! Considering particularism in research design and reporting", Journal of Information Technology 31(3), 2015, pp. 241249.

[12] DePietro, R., E. Wiarda, and M. Fleischer, "The context for change: Organization, technology and environment", In L. Tornatzky and M. Fleischer, eds., The Process of Technological Innovation. Lexington Books, Lexington, USA, 1990, 152-175.

[13] European Parliament, Regulation (EU) 2016/679 of the european parliament and of the council, 2016.

[14] FDA, Proposed regulatory framework for modifications to artificial intelligence/Machine learning (AI/ML)-based software as a medical device (SaMD)-discussion paper and request for feedback, 2019.

[15] Flick, U., "Triangulation in qualitative research", In U. Flick, E. von Kardoff and I. Steinke, eds., A Companion to Qualitative Research. Sage, London, UK, 2004, 178-183.

[16] Greener, S., Business research methods, Ventus Publishing, London, UK, 2008.

[17] Greenhalgh, T., J. Wherton, C. Papoutsi, et al., "Beyond adoption: A new framework for theorizing and evaluating nonadoption, abandonment, and challenges to the scale-up, spread, and sustainability of health and care technologies", Journal of Medical Internet Research 19(11), 2017.

[18] He, J., S.L. Baxter, J. Xu, J. Xu, X. Zhou, and K. Zhang, "The practical implementation of artificial intelligence technologies in medicine", Nature Medicine 25(1), 2019, pp. 30-36.

[19] Hofmann, P., P. Rust, and N. Urbach, "Machine learning approaches along the radiology value chain - Rethinking value propositions.", Proceedings of the 27th European Conference on Information Systems, (2019).

[20] Hsieh, H.-F., and S.E. Shannon, "Three approaches to 
qualitative content analysis", Qualitative Health Research 15(9), 2005, pp. 1277-1288.

[21] Hufnagl, C., E. Doctor, L. Behrens, C. Buck, and T. Eymann, "Digitisation along the patient pathway in hospitals", Proceedings of the 27th European Conference on Information Systems, (2019).

[22] Hung, S.-Y., W.-H. Hung, C.-A. Tsai, and S.-C. Jiang, "Critical factors of hospital adoption on CRM system: Organizational and information system perspectives", Decision Support Systems 48(4), 2010, pp. 592-603.

[23] Jordan, M.I., and T.M. Mitchell, "Machine learning: Trends, perspectives, and prospects", Science 349(6245), 2015, pp. 255-260.

[24] Kuan, R., "Adopting AI in health care will be slow and difficult”, Harvard Business Review Digital Articles, 2019.

[25] Lebovitz, S., "Diagnostic doubt and artificial intelligence: An inductive field study of radiology work", Proceedings of the 40th International Conference on Information Systems, (2019).

[26] Lee, J., C. Liu, N. Shang, et al., Generate the concept representation using OMOP ontology graph, New York, USA, 2019.

[27] Li, T., Y. Zhang, C. Gong, et al., "Prevalence of malnutrition and analysis of related factors in elderly patients with COVID-19 in Wuhan, China", European Journal of Clinical Nutrition, 2020.

[28] Lian, J.W., D.C. Yen, and Y.T. Wang, “An exploratory study to understand the critical factors affecting the decision to adopt cloud computing in Taiwan hospital", International Journal of Information Management 34(1), 2014, pp. 28-36.

[29] Liu, D., N. Sepulveda, and M. Zheng, “Artificial neural networks condensation: A strategy to facilitate adaption of machine learning in medical settings by reducing computational burden", ArXiv, 2018.

[30] Mayer, K.J.R., and R.T. Sparrowe, "From the editors integrating theories in AMJ articles", Academy of Management Journal 56(4), 2013, pp. 917-922.

[31] McCarthy, J., What is Artificial Intelligence?, Stanford, 2007.

[32] Migliore, A., "On the new regulation of medical devices in europe", Expert Review of Medical Devices 14(12), 2017, pp. 921-923.

[33] Myers, M.D., and M. Newman, "The qualitative interview in IS research: Examining the craft", Information and Organization 17(1), 2007, pp. 2-26.

[34] Panch, T., H. Mattie, and L.A. Celi, "The "inconvenient truth' about AI in healthcare", npj Digital Medicine 2(1), 2019, pp. 4-6.

[35] Paton, C., and S. Kobayashi, "An open science approach to artificial intelligence in healthcare", Yearbook of medical informatics 28(1), 2019, pp. 47-51.

[36] Prasser, F., F. Kohlmayer, R. Lautenschläger, and K.A.
Kuhn, “ARX - A comprehensive tool for anonymizing biomedical data", Proceedings of Annual Symposium American Medical Informatics Association, (2014), 984-993.

[37] Pumplun, L., and P. Buxmann, "Intelligent Systems and Hospitals: Joint Forces in the Name of Health?", Proceedings of the 15th International Conference on Wirtschaftsinformatik, (2020).

[38] Rai, A., and S. Sarker, "Editor's comments: Nextgeneration digital platforms: Toward human-AI hybrids", MIS Quarterly 43(1), 2019, pp. iii-ix.

[39] Russell, S.J., and P. Norvig, Artificial intelligence: A modern approach, Addison Wesley, Boston, MA, USA, 2016.

[40] Saldaña, J., The coding manual for qualitative researchers, Sage, London, UK, 2009.

[41] Shahid, N., T. Rappon, and W. Berta, “Applications of artificial neural networks in health care organizational decision-making: A scoping review", PLOS ONE 14(2), 2019, pp. 1-22.

[42] Shaw, J., F. Rudzicz, T. Jamieson, and A. Goldfarb, "Artificial intelligence and the implementation challenge", Journal of Medical Internet Research 21(7), 2019.

[43] Thrall, J.H., X. Li, Q. Li, et al., "Artificial intelligence and machine learning in radiology: Opportunities, challenges, pitfalls, and criteria for success.", Journal of the American College of Radiology 15(3), 2018, pp. 504-508.

[44] Topol, E.J., "High-performance medicine: the convergence of human and artificial intelligence", Nature Medicine 25(1), 2019, pp. 44-56.

[45] Tornatzky, L.G., and K.J. Klein, "Innovation characteristics and innovation adoption-implementation: A meta-analysis of findings.", IEEE Transactions on Engineering Management EM-29(1), 1982, pp. 28-45.

[46] Vardell, E.J., and M. Moore, "Isabel, a clinical decision support system”, Medical Reference Services Quarterly 30(2), 2011, pp. 158-166.

[47] Yang, Z., J. Sun, Y. Zhang, and Y. Wang, "Understanding SaaS adoption from the perspective of organizational users: A tripod readiness model", Computers in Human Behavior 45, 2015, pp. 254-264.

[48] Zhang, Y., M. Qiu, C.W. Tsai, M.M. Hassan, and A. Alamri, "Health-CPS: Healthcare cyber-physical system assisted by cloud and big data", IEEE Systems Journal 11(1), 2017, pp. 88-95.

[49] Zhu, K., and K.L. Kraemer, "Post-adoption variations in usage and value of e-business by organizations: Crosscountry evidence from the retail industry", Information Systems Research 16(1), 2005, pp. 61-84.

[50] Zhu, K., K.L. Kraemer, and S. Xu, "The process of innovation assimilation by firms in different countries: A technology diffusion perspective on e-business", Management Science 52(10), 2006, pp. 1557-1576. 